\title{
PRÁTICAS DE ECONOMIA CIRCULAR NO DESENVOLVIMENTO DE PRODUTOS AERONÁUTICOS: AÇÕES PROPOSTAS PELO MODELO RESOLVE
}

Veruska Mazza Rodrigues Dias (veve_mazza@ hotmail.com) - Departamento de Engenharia de Produção / Faculdade de Engenharia / Universidade Estadual Paulista "Júlio de Mesquita Filho" Daniel Jugend (aniel@feb.unesp.br) - Departamento de Engenharia de Produção / Faculdade de Engenharia / Universidade Estadual Paulista "Júlio de Mesquita Filho"

Carlos Razzino (carlos.razzino@unesp.br) - Departamento de Engenharia de Produção / Faculdade de Engenharia / Universidade Estadual Paulista “Júlio de Mesquita Filho"

\section{RESUMO}

A preocupação com a adoção da sustentabilidade ambiental vem atraindo cada vez mais a atenção de praticantes e pesquisadores. Nos últimos anos a abordagem da economia circular tem se destacado como uma das principais tendências em sustentabilidade ambiental. Esta pesquisa, por meio de revisão teórica sistemática, visa propor framework integrando características de economia circular, definidas pelas ações propostas no modelo ReSOLVE às atividades de desenvolvimento de novos produtos e, especialmente, considerando as características dos produtos aeronáuticos. Como resultados, espera-se que o framework proposto inicie discussões sobre a integração da economia circular no desenvolvimento de novos produtos, considerando, especialmente, as características de produtos aeronáuticos.

Palavras chave: economia circular; modelo ReSOLVE; desenvolvimento de produtos ambientalmente sustentáveis; desenvolvimento de produtos aeronáuticos. 


\section{INTRODUÇÃ̃}

A introdução de processos de produção mais sustentáveis, sob o ponto de vista ambiental, nas indústrias está aumentando rapidamente em todo o mundo (HALLSTEDT et al., 2015). Tem chamado mais atenção dos consumidores a cada dia, a necessidade de produtos e bens sustentáveis e, especialmente, que respeitem o meio ambiente. É importante que os fatores de sustentabilidade sejam considerados no processo de desenvolvimento de produtos (PDP), desde os estágios preliminares de design do produto e analisados de forma a estarem integrados ao mesmo durante todo seu ciclo de vida (matérias-primas, produção, distribuição, uso, manutenção e fim de vida) (GIALOS et al., 2018).

A preocupação com a sustentabilidade e implantação da cultura verde nas empresas, tanto a níveis estratégicos como operacionais, vem sendo foco de atuais pesquisas em gestão de operações (ZENG, FU E OUYANG, 2018; ABSON et al., 2017). Em uma análise de viabilidade sobre a implantação do conceito de sustentabilidade ambiental nos produtos, verifica-se que, em curto prazo, tal prática pode aumentar o custo (custo do material, custo de fabricação, custo de teste e avaliação, etc.) em alguns casos; no entanto, no longo prazo eles podem gerar impacto positivo no desempenho financeiro e inovador das firmas (GIALOS et al., 2018).

Empresas que fabricam produtos que são produzidos durante um longo período de tempo e precisam ser apoiados ou mantidos por muito mais tempo após o término do processo de produção, podem alcançar os benefícios de custo em longo prazo ao integrarem a sustentabilidade ambiental desde as etapas de projeto dos produtos (GIALOS et al., 2018). A indústria aeronáutica se enquadra neste perfil, visto que seus produtos têm um ciclo de vida médio de mais de 20 anos, o que torna essencial se ampliarem as pesquisas sobre desenvolvimento de produtos ambientalmente sustentáveis nesta indústria.

Outro grande fator motivador, econômico e social, que evidencia as necessidades de pesquisa sobre a adoção de práticas sustentáveis na aviação como, por exemplo, no processo de desenvolvimento de produtos aeronáuticos, é o de que com a globalização e com o rápido crescimento da exploração comercial do transporte aeroespacial, as projeções para o mercado global aeronáutico indicam que o uso deste meio triplicará até 2050. Em termos de passageiros, este número dobrou nos últimos 15 anos e a perspectiva é que dobre novamente nos próximos 15 anos (HALLSTEDT et al., 2015). 
Assim, por meio de revisão teórica sistemática, esse artigo tem por objetivo contribuir para a integração da economia circular ao desenvolvimento de produtos aeronáuticos ao propor framework que integre essas duas áreas. Além disso, esse trabalho visa também responder a seguinte questão: Quais são as práticas de economia circular que as empresas aeronáuticas poderiam utilizar no desenvolvimento de novos produtos?

Para isso, inicialmente apresenta-se revisão teórica sobre o tema. Posteriormente, o método de pesquisa é explicado e os resultados são apresentados. Por fim, são delineadas as discussões e considerações finais.

\section{REVISÃO TEÓRICA}

\subsection{Economia Circular - Modelo ReSOLVE}

A economia circular oferece diversos mecanismos de criação de valor dissociados do consumo de recursos finitos. As vantagens importantes do sistema de economia circular sobre a economia convencional (linear) têm motivado muitos estudos, sendo que estes vêm se tornando evidentes na literatura nos últimos anos. Estes estudos examinam diferentes perspectivas da economia circular dentro de uma ampla gama de aplicações como, por exemplo, manufatura (LIEDER E RASHID, 2016), serviço (TUKKER, 2015), construção (SMOL et al., 2015) e cadeia de suprimentos (ZHU, GENG E LAI, 2010).

Por meio de pesquisa realizada em estudos de caso e entrevistas com especialistas, a Fundação Ellen MacArthur (2015) identificou um conjunto de seis ações que empresas e governos podem adotar visando à transição para a economia circular: regenerar, compartilhar, otimizar, ciclar, virtualizar e trocar. Juntas, essas ações formam a estrutura ReSOLVE. A estrutura ReSOLVE proporciona a empresas e governos uma ferramenta para a geração de estratégias circulares e iniciativas voltadas para o crescimento. De maneiras diferentes, essas ações aumentam a utilização de ativos físicos, prolongam sua vida e promovem a substituição do uso de recursos finitos pelo de fontes renováveis. Cada ação reforça e acelera o desempenho das outras. Essas ações são usadas como base na definição das práticas aplicadas nas empresas para a transição de um modelo econômico linear a um modelo circular (EMF, 2015; EMF, 2017).

Para que a seja possível a inserção desse novo viés do processo de PDP, de forma bemsucedida, é preciso que as empresas sejam capazes de adaptarem-se de forma satisfatória, sendo 
necessárias otimizações na cadeia de suprimentos, no design de produtos, na seleção de matérias primas, nos processos de produção e em sua entrega aos consumidores, conforme observaram trabalhos como o de Linton, Klassen e Jayaraman (2007) e Darnall, Jolley e Handfield (2008), o que reforça o grau de importância de pesquisas sobre o desenvolvimento de novos produtos, de modo a integrar também práticas consideradas "verdes" neste processo.

\subsection{Sustentabilidade no Desenvolvimento de Produtos Aeronáuticos}

Nas dimensões atuais, o setor aeronáutico já se destaca como o segundo maior consumidor mundial de combustível para transporte. Cerca de 250 milhões de toneladas de óleo ao ano, representando $12 \%$ da energia consumida pelo setor de transporte e $9 \%$ da emissão de gases de efeito estufa (GHG) (HUANG et al., 2016) do mundo. Portanto, é relevante o desenvolvimento de novos produtos com características de ecodesign, produtos otimizados e ecologicamente mais eficientes que permitam a redução de gasto energético e emissões de GHG (IMMARIGEON et al., 1995), o que é alinhado com os objetivos propostos pelo conceito de economia circular. Simultaneamente, observa-se que a oferta dos produtos que buscam minimizar impactos ao meio ambiente tem sido valorizada (JI, MA E LI, 2015; KIRCHOFF, KOCH E SATINOVER NICHOLS, 2011) e que os padrões de consumo e de produção têm apresentado significativas mudanças ao longo das últimas décadas (SMITH E OFFODILE, 2016).

A integração desses dois cenários tem feito com que as grandes fabricantes de aeronaves e componentes como Airbus, Boeing e General Electric repensassem seu processo de manufatura quanto à necessidade de evolução do processo convencional para um processo de manufatura sustentável, vendo essa transição como uma oportunidade de negócio (HALLSTEDT et al., 2015) e viabilizando essa estratégia através da implantação de materiais primários alternativos e processos inovadores de fabricação que permitam a redução da elevada razão "buy-to-fly", ou seja, a massa de matéria-prima necessária por unidade de massa de componente acabado (ALLWOOD, ASHBY, GUTOWSKI E WORRELL, 2011). Porém, ainda são tímidas as iniciativas para se implantar a manufatura sustentável na indústria aeronáutica e, apenas alguns casos isolados são conhecidos. 


\section{MÉTODO DE PESQUISA}

Foram realizados acessos ao banco de dados internacional Scopus e de algumas das mais importantes revistas em Engenharia de Produção, Sustentabilidade, Aeronáutica e Aeroespacial para identificação de artigos científicos acerca dos temas economia circular, modelo ReSOLVE, desenvolvimento de produtos aeronáuticos e manufatura de aeronaves. Esta pesquisa foi realizada em duas etapas. A primeira teve por objetivo identificar a relevância atual do tema central desta pesquisa, economia circular, observando-se a fase de maturidade do mesmo quanto ao número de publicações, ou seja, se em ascensão ou decadência para que fosse analisada a viabilidade em pesquisar-se sobre o assunto.

A busca, atualizada em 27/03/2019, sobre o termo "circular economy", foi realizada sem restrição de período ou acesso, porém, restrita sobre o tipo do documento de interesse, onde foram considerados relevantes para essa pesquisa artigos publicados e artigos in press. Para essas categorias foram encontrados 1973 e 86 documentos, respectivamente, totalizando 2059 documentos de interesse.

Já na segunda fase da pesquisa, foram realizadas outras duas buscas sendo que, a primeira delas, fazia integração direta entre os temas economia circular e a indústria aeroespacial. Ao todo, foram identificados durante esta fase da revisão teórica 6 documentos. Devido à baixa quantidade de documentos encontrados, efetuou-se uma segunda busca ampliando-se o conceito de economia circular para o tema sustentabilidade, com o objetivo de identificar-se o viés de sustentabilidade da indústria aeroespacial e realizar a avaliação histórica sobre o aumento ou diminuição de pesquisas sobre o tema. Ao todo, foram identificados durante esta fase da revisão teórica 459 documentos.

$\mathrm{Na}$ análise de histórico de publicações sobre economia circular, correspondente à primeira fase dessa pesquisa, nota-se um crescimento abrupto de pesquisas nessa área a partir de 2015, sendo que o crescimento se mantém ao longo dos últimos anos, a partir daquela data. Durante a segunda fase da pesquisa é possível identificar crescimento significativo do interesse pelo tema sustentabilidade a partir de 2005 e, crescimento ininterrupto desde 2015. Dessa forma, esse foi considerado o período de interesse sendo feito um recorte temporal de 5 anos (2015-2019) que reduziu o número de documentos para 239 documentos. Dos artigos encontrados foram lidos os resumos para avaliação do conteúdo e relevância para essa pesquisa. De acordo com o tema de interesse, foram classificados como parte da revisão teórica 110 
desses artigos. Além dos documentos resultantes do processo de busca, foram acrescentados artigos considerados relevantes pelo autor que envolvessem um dos temas de interesse dessa pesquisa e não a intersecção dos temas (resultado das buscas). Assim os documentos selecionados, foram usados como base para a composição do framework.

Observando-se os resultados encontrados, foi possível verificar que o tema de desenvolvimento/produção sustentável na indústria aeronáutica vem sendo a cada vez mais investigado, porém, no contexto micro de economia circular, o assunto é pouco amadurecido, o que reforça o interesse em pesquisar-se sobre o tema. Após a conclusão das buscas, iniciouse a leitura dos artigos e a construção do framework teórico, sendo o modelo ReSOLVE adotado como base.

Primeiramente, foram investigadas práticas teóricas de economia circular nos artigos encontrados durante a busca e tais práticas foram relacionadas às seis ações que empresas e governos podem adotar visando à transição para a economia circular. Em seguida, foram levantadas, também na literatura, as práticas adotadas na aeronáutica que se relacionam com àquelas encontradas na literatura para o tema central, economia circular.

Com base nas informações adquiridas por meio do método apresentado, a proposta desse trabalho foi desenvolver um framework teórico que incorpore características de economia circular, definidas pelas ações propostas no modelo ReSOLVE, às atividades de desenvolvimento de produtos aeronáuticos.

\section{RESULTADOS}

As figuras 1 a 6 apresentam os resultados deste trabalho, isto é, a forma como cada uma das ações do modelo ReSOLVE podem ser adotadas considerando as necessidades do desenvolvimento de produtos aeronáuticos. A implantação de práticas sustentáveis na indústria aeronáutica foi analisada através dos resultados encontrados durante a revisão sistemática e cada uma dessas iniciativas foi, então, relacionada a uma prática de economia circular, assim como, aos conceitos propostos pelo modelo ReSOLVE. 


\begin{tabular}{|c|c|c|c|c|}
\hline $\begin{array}{l}\text { Objetivo Circular } \\
\text { modelo ReSOLVE } \\
\text { (ações) }\end{array}$ & $\begin{array}{c}\text { Estratégia } \\
\text { (práticas de EC) }\end{array}$ & Autores & $\begin{array}{l}\text { Exemplo de Iniciativa } \\
\text { (práticas na aeronáutica) }\end{array}$ & Autores \\
\hline $\begin{array}{l}\text { Regenerar } \\
\text { - Mudança para energia } \\
\text { renovável e materiais } \\
\text { - Recuperar, reter e } \\
\text { restaurar a saúde dos } \\
\text { ecossistemas } \\
\text { - Retomo dos recursos } \\
\text { biológicos recuperados ao } \\
\text { biosfera }\end{array}$ & $\begin{array}{l}\text { - Uso de materiais de base biológica } \\
\text { - Uso de materiais ambientalmente } \\
\text { amigáveis } \\
\text { - Restauração: também conhecido } \\
\text { como compostagem } \\
\text { - Redução das emissões de GEE } \\
\text { - Melhoria da qualidade do ar } \\
\text { - Eliminação de substâncias que } \\
\text { empobrecem a camada de ozônio } \\
\text { - Redução da emissão de ruído } \\
\text { - Retirada do produto (aeronaves) } \\
\text { após o fim da vida útill fim de uso e } \\
\text { atualização da remanufatura' } \\
\text { reutilização/ reforma }\end{array}$ & $\begin{array}{l}\text { KALMYKOVA, SADAGOPAN E } \\
\text { ROSADO, } 2018 \\
\text { ACCORSI et al., } 2014 \\
\text { AHI E SEARCY, } 2015 \\
\text { ZHU E SARKIS, } 2004 \\
\text { GOPALAKRISHNAN et al., } 2012 \\
\text { KALMYKOVA, SADAGOPAN E } \\
\text { ROSADO, 2018 } \\
\text { VARSEI E POLYAKOVSKIY, } \\
\text { 2017 } \\
\text { ZHANG E XIE, 2014 } \\
\text { REEFKE E SUNDARAM, } 2017 \\
\text { VACHON E KL.ASSEN, } 2008 \\
\text { BOUKHERROUB et al,, } 2015 \\
\text { GRI, 2013 } \\
\text { GOPALAKRISHNAN et al., } 2012 \\
\text { COWPER-SMITH E DE } \\
\text { GROSBOIS, 2011 } \\
\text { CETINKAYA et al., } 2011 \\
\text { AHI E SEARCY, } 2015 \\
\text { EROL, SENCER E SARI, } 2011 \\
\text { KEIVANPOUR, AIT KADI E } \\
\text { MASCLE, 2015 }\end{array}$ & 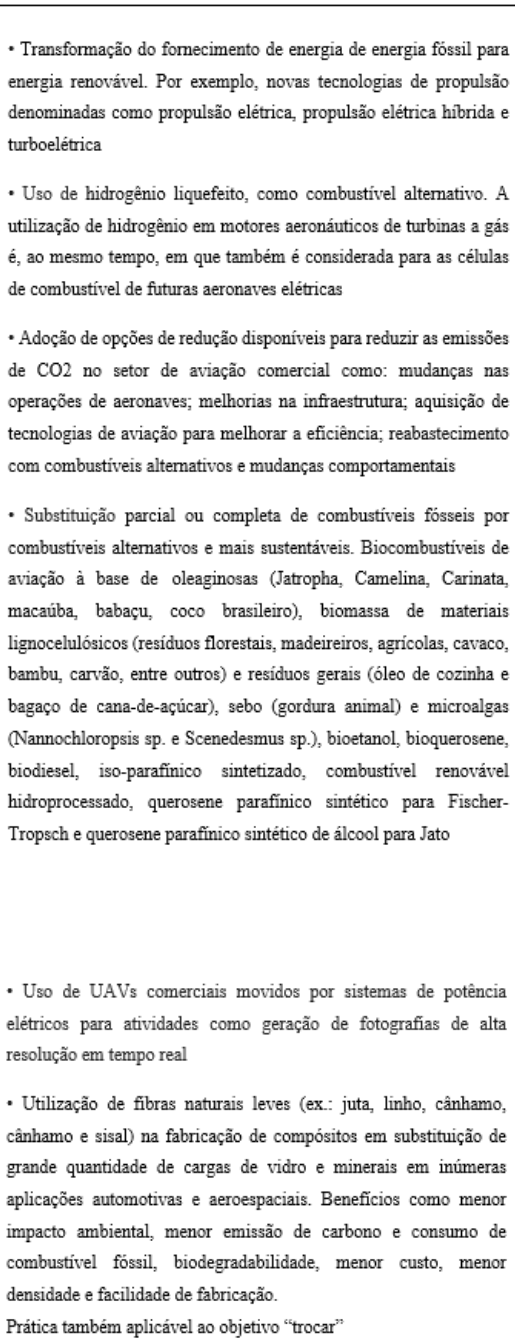 & $\begin{array}{l}\text { BWAPWA, ANANDRAJ E TROIS, } \\
2018 \\
\text { BESSA et al., } 2019 \\
\text { BESSETTE et al., } 2018 \\
\text { DINZ, SARGEANT E MILLAR, } \\
2018 \\
\text { DODD, ORLITZKY E NELSON, } \\
2018 \\
\text { MICHAILOS, } 2017 \\
\text { RAIIAN E KOT, } 2018 \\
\text { RASSOULINEJAD-MOUSAVI, } \\
\text { MAO E ZHANG, 2018 } \\
\text { CAVALETT E CHERUBIN, } 2018 \\
\text { CHEN E REN, } 2018 \\
\text { SÖGÜT, } 2018 \\
\\
\text { PARK, LEE E CHON, } 2019\end{array}$ \\
\hline
\end{tabular}

FIGURA 1 - Ação Regenerar. Fonte: Autor (2019)

\begin{tabular}{|c|c|c|c|c|}
\hline $\begin{array}{c}\text { Objetivo Circular } \\
\text { modelo ReSOLVE } \\
\text { (ações) }\end{array}$ & $\begin{array}{c}\text { Estratégia } \\
\text { (práticas de } \mathrm{EC} \text { ) }\end{array}$ & Autores & $\begin{array}{l}\text { Exemplo de Iniciativa } \\
\text { (práticas na aeronáutica) }\end{array}$ & Autores \\
\hline $\begin{array}{l}\text { Compartilhar } \\
\text { - Compartilhar ativos (por } \\
\text { exemplo, carros, salas, } \\
\text { eletrodomésticos) } \\
\text { - Reutilização / segunda } \\
\text { mão } \\
\text { - Prolongar a vida através } \\
\text { da manutenção, design para } \\
\text { durabilidade, capacidade de } \\
\text { atualização, etc. }\end{array}$ & $\begin{array}{l}\text { - Simbiose industrial } \\
\text { - Espaço polivalente } \\
\text { - Reforma, remanufatura e } \\
\text { recondicionamento de produtos } \\
\text { - Uso de materiais de sucata para } \\
\text { subprodutos. } \\
\text { Estratégia também aplicável ao } \\
\text { objetivo "ciclar" } \\
\text { - Reutilização secundária }\end{array}$ & $\begin{array}{l}\text { KALMYKOVA, } \\
\text { SADAGOPAN E ROSADO, } \\
2018 \\
\text { KALMYKOVA, } \\
\text { SADAGOPAN E ROSADO, } \\
2018 \\
\text { KALMYKOVA, } \\
\text { SADAGOPAN E ROSADO, } \\
2018 \\
\text { RAJE SRIVASTAVA, } 2018 \\
\text { KALMYKOVA, } \\
\text { SADAGOPAN E ROSADO, } \\
\text { 2018 }\end{array}$ & $\begin{array}{l}\text { - Uso de compósitos reciclados em estruturas não-críticas de } \\
\text { aeronaves, como galeras, revestimentos intemos, peças de assentos } \\
\text { e ferramentas que produzam materiais mais resistentes e leves no } \\
\text { mesmo setor. Prática também aplicável ao objetivo "ciclar" } \\
\text { - Desmontagem de aeronaves para reutilização de materiais e } \\
\text { componentes como peças de reposição de alta qualidade. Prática } \\
\text { também aplicável ao objetivo "ciclar" } \\
\text { - Venda de peças de aeronaves para uso como mobiliário de } \\
\text { escritório e para fins decorativos. Prática também aplicável ao } \\
\text { objetivo "ciclar" }\end{array}$ & $\begin{array}{l}\text { ASMATULU, OVERCASH E } \\
\text { TWOMEY, } 2013 \\
\text { ASMATULU, OVERCASH E } \\
\text { TWOMEY, } 2013 \\
\text { ASMATULU, OVERCASH E } \\
\text { TWOMEY, } 2013\end{array}$ \\
\hline
\end{tabular}




\begin{tabular}{|l|l|} 
- Agua reciclada e reutilizada & REEFKE E SUNDARAM, 2017 \\
$\begin{array}{l}\text { Estratégia também aplicável aos } \\
\text { objetivos "otimizar" e "ciclar" }\end{array}$ & \\
$\begin{array}{l}\text { - Design de produtos apreciável e e } \\
\text { durável }\end{array}$ & $\begin{array}{l}\text { GOVINDAN E HASANAGIC, } \\
2018 \\
\text { - Atualização, manutenção e reparo } \\
\text { de produtos }\end{array}$ \\
$\begin{array}{ll}\text { KALMYKOVA, } \\
\text { SADAGOPAN E ROSADO, } \\
\text { Realização de avaliação do ciclo de } \\
\text { vida de todos os produtos }\end{array}$ & $\begin{array}{l}\text { GOPALAKRISHNAN et al., } \\
\text { 2012 }\end{array}$
\end{tabular}

FIGURA 2 - Ação Compartilhar. Fonte: Autor (2019)

\begin{tabular}{|c|c|c|c|c|}
\hline $\begin{array}{c}\text { Objetivo Circular } \\
\text { modelo ReSOLVE } \\
\text { (ações) }\end{array}$ & $\begin{array}{c}\text { Estratégia } \\
\text { (práticas de } \mathrm{EC} \text { ) }\end{array}$ & Autores & $\begin{array}{l}\text { Exemplo de Iniciativa } \\
\text { (práticas na aeronáutica) }\end{array}$ & Autores \\
\hline $\begin{array}{l}\text { Otimizar } \\
\text { - Aumentar o desempenho/ } \\
\text { eficiência do produto } \\
\text { - Remover resíduos na } \\
\text { produção e cadeia de } \\
\text { suprimentos } \\
\text { - Alavancar big data, } \\
\text { automação, sensoriamento } \\
\text { remoto e direção }\end{array}$ & $\begin{array}{l}\text { Diversidade e vínculos } \\
\text { intersetoriais } \\
\text { - Mitigação do impacto dos } \\
\text { impactos ambientais de produtos e } \\
\text { serviços } \\
\text { - Design de embalagem otimizado } \\
\text { - Consumo eficiente de energia na } \\
\text { fabricação e serviços } \\
\text { - Controle da intensidade energética } \\
\text { - Auditoria energética } \\
\text { - Liberação de efluentes e poluentes } \\
\text { de água } \\
\text { - Projeto e fabricação envolvendo } \\
\text { redução } \\
\text { - Aumento da ecoeficiência na } \\
\text { produção } \\
\text { - Uso efíciente da água na fabricação } \\
\text { e serviços } \\
\text { - Segregação de resíduos e sistema } \\
\text { adequado de descarte de resíduos } \\
\text { - Compras mais limpas } \\
\text { - Colaboração com fornecedores e } \\
\text { seleção de novos formecedores } \\
\text { (desenvolvimento) usando critérios } \\
\text { ambientais } \\
\text { - Sistema seguro de eliminação de } \\
\text { lixo eletrônico } \\
\text { - Sistema de informação eficiente }\end{array}$ & 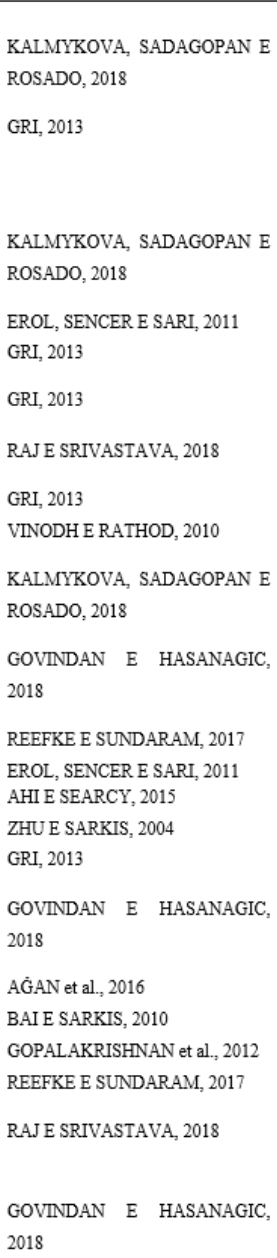 & $\begin{array}{l}\text { - Projeto de veículos de massa reduzida (a versão leve de um veículo } \\
\text { consome menos combustivel do que sua contraparte mais pesada na } \\
\text { mesma distância) orientado a reduzir o consumo de combustivel } \\
\text { (FC) e as emissões de carbono resultantes. Uso de ferramentas de } \\
\text { modelagem computacional para simular o impacto das reduções de } \\
\text { massa nos fatores de desempenho do veículo } \\
\text { - Projeto ou melhoria dos sistemas de produção de energia, incluindo } \\
\text { motorizações convencionais mais eficientes e motores avançados, } \\
\text { com ênfase especial na redução da perda de energia e consequente } \\
\text { aumento da eficiência em termos de consumo de combustivel } \\
\text { - Substituição dos processos de fabricação convencionais, tais como } \\
\text { o processo de rebitagem, por processos de aditivo inovadores, tais } \\
\text { como o processo de feixe de laser de soldadura (LBW), a fim de } \\
\text { reduzir a elevada razão "buy-to-fly" (a massa de matéria-prima } \\
\text { necessária por massa unitária do componente acabado) e produzir } \\
\text { aeronaves mais leves, consequentemente, redução de uso de energia } \\
\text { e emissões de gases de efeito estufa (GEE) } \\
\text { - Gestão (redução de resíduos, reutilização, reciclagem, recuperação } \\
\text { e aterro) de resíduos compostos gerados durante a fabricação de } \\
\text { peças compostas aeroespaciais - plásticos reforçados com fibra de } \\
\text { carbono (CFRP). Prática também aplicável ao objetivo "ciclar" } \\
\text { - Gestão de resíduos aeroportuários produzidos em escritórios, } \\
\text { pontos de venda, restaurantes, banheiros e restaurantes de voo, de } \\
\text { operações de carga aérea, instalações de manutenção e áreas e de } \\
\text { projetos de paisagismo, construção e demolição realizados no } \\
\text { aeroporto. Por exemplo: recipientes de alimentos e bebidas, jomais } \\
\text { e revistas, restos de comida (de salões/ cafeterias), lâmpadas, toner } \\
\text { de impressora, papel, documentos e impressões de computador - } \\
\text { gerados por companhias aéreas } \\
\text { - Algoritmo de automação do PDP (análise de lead time, custo de } \\
\text { processo e custo de investimento) implementado no processo de } \\
\text { projeto conceitual das conexões de articulação de um conjunto de } \\
\text { leme no estabilizador vertical de um jato executivo } \\
\text { - Algoritmo sobre a estratégia de desmontagem de peças mecânicas } \\
\text { em aeronaves }\end{array}$ & $\begin{array}{l}\text { MULDER, LA ROCCA, } \\
\text { SCHUT E VERHAGEN, } 2015 \\
\text { ASMATULU, OVERCASH E } \\
\text { TWOMEY, 2013 }\end{array}$ \\
\hline
\end{tabular}

FIGURA 3 - Ação Otimizar. Fonte: Autor (2019) 


\begin{tabular}{|c|c|c|c|c|}
\hline $\begin{array}{c}\text { Objetivo Circular } \\
\text { modelo ReSOLVE } \\
\text { (ações) }\end{array}$ & $\begin{array}{c}\text { Estratégia } \\
\text { (práticas de } \mathrm{EC} \text { ) }\end{array}$ & Autores & $\begin{array}{l}\text { Exemplo de Iniciativa } \\
\text { (práticas na aeronáutica) }\end{array}$ & Autores \\
\hline $\begin{array}{l}\text { Ciclar } \\
\text { - Produtos ou componentes } \\
\text { remanufaturados } \\
\text { - Reciclar materiais } \\
\text { - Digerir anaerobicamente } \\
\text { - Extrair compostos } \\
\text { bioquímicos de resíduos } \\
\text { orgânicos }\end{array}$ & $\begin{array}{l}\text { - Design para desmontagem/ } \\
\text { reciclagem } \\
\text { - Reforma/ reparo/ remanufatura de } \\
\text { produtos } \\
\text { - Sistemas de devolução e troca } \\
\text { - Rede ecoindustrial regional mais } \\
\text { fácil } \\
\text { - Equipamentos técnicos e } \\
\text { instalações para remanufatura } \\
\text { - Introdução de reclassificação na } \\
\text { produção } \\
\text { - Reciclagem funcional } \\
\text { - Extração de bioquimicos } \\
\text { Reciclagem de alta qualidade }\end{array}$ & $\begin{array}{l}\text { KALMYKOVA, } \\
\text { SADAGOPAN E ROSADO, } \\
2018 \\
\text { KALMYKOVA, } \\
\text { SADAGOPAN E ROSADO, } \\
2018 \\
\text { KALMYKOVA, } \\
\text { SADAGOPAN E ROSADO, } \\
2018 \\
\text { GOVINDAN E HASANAGIC, } \\
2018 \\
\text { GOVINDAN E HASANAGIC, } \\
2018 \\
\text { GOVINDAN E HASANAGIC, } \\
2018 \\
\text { KALMYKOVA, } \\
\text { SADAGOPAN E ROSADO, } \\
2018 \\
\text { KALMYKOVA, } \\
\text { SADAGOPAN E ROSADO, } \\
2018 \\
\text { KALMYKOVA, } \\
\text { SADAGOPAN E ROSADO, } \\
\text { 2018 }\end{array}$ & $\begin{array}{l}\text { - Reciclagem de fibra de carbono de compósitos } \\
\text { - Uso de compósitos reciclados em estruturas não-críticas de } \\
\text { aeronaves, como galeras, revestimentos intermos, peças de assentos } \\
\text { e ferramentas que produzam materiais mais resistentes e leves no } \\
\text { mesmo setor } \\
\text { - Desmontagem de aeronaves para reutilização de materiais e } \\
\text { componentes como peças de reposição de alta qualidade } \\
\text { - Venda de peças de aeronaves para uso como mobiliário de } \\
\text { escritório e para fins decorativos }\end{array}$ & $\begin{array}{l}\text { ASMATULU, OVERCASH E } \\
\text { TWOMEY, } 2013 \\
\text { ASMATULU, OVERCASH E } \\
\text { TWOMEY, } 2013 \\
\\
\text { ASMATULU, OVERCASH E } \\
\text { TWOMEY, } 2013 \\
\text { ASMATULU, OVERCASH E } \\
\text { TWOMEY, } 2013\end{array}$ \\
\hline
\end{tabular}

FIGURA 4 - Ação Ciclar. Fonte: Autor (2019)

\begin{tabular}{|c|c|c|c|c|}
\hline $\begin{array}{c}\text { Objetivo Circular } \\
\text { modelo ReSOLVE } \\
\text { (ações) }\end{array}$ & $\begin{array}{c}\text { Estratégia } \\
\text { (práticas de EC) }\end{array}$ & Autores & $\begin{array}{l}\text { Exemplo de Iniciativa } \\
\text { (práticas na aeronáutica) }\end{array}$ & Autores \\
\hline $\begin{array}{l}\text { Virtualizar } \\
\text { diretamente (por exemplo, } \\
\text { livros, CDs,DVDs, viagens) } \\
\text { - Desmaterializar } \\
\text { indiretamente (por exemplo, } \\
\text { compras on-line) }\end{array}$ & $\begin{array}{l}\text { - Virtualizar/ Desmaterializar: por } \\
\text { exemplo, livros eletrônicos/CDs, } \\
\text { compras on-line, uso de } \\
\text { telecomunicações para diminuir o } \\
\text { uso de escritórios e viagens }\end{array}$ & $\begin{array}{l}\text { KALMYKOVA, } \\
\text { SADAGOPAN E ROSADO, } \\
2018\end{array}$ & $\begin{array}{l}\text { - Aplicação do conceito de TI verde, através de práticas como e- } \\
\text { commerce, edifícios inteligentes, inteligência artificial e sistemas de } \\
\text { transporte inteligentes. TI verde definido como a integração e } \\
\text { cooperação de projetar, fabricar, utilizar e descartar de forma } \\
\text { eficiente e efetiva computadores, servidores e outros subsistemas } \\
\text { aassociados, visando à prevenção da poluição, manejo de produtos e } \\
\text { desenvolvimento sustentável, e concentrando-se fortemente no uso } \\
\text { de sistemas de informação para aprimorar sustentabilidade em toda } \\
\text { a economia }\end{array}$ & ZENG, FU E OUYANG, 2018 \\
\hline
\end{tabular}

FIGURA 5 - Ação Virtualizar. Fonte: Autor (2019) 


\begin{tabular}{|c|c|c|c|c|}
\hline $\begin{array}{c}\text { Objetivo Circular } \\
\text { modelo ReSOL VE (ações) }\end{array}$ & $\begin{array}{c}\text { Estratégia } \\
\text { (práticas de EC) }\end{array}$ & Autores & $\begin{array}{l}\text { Exemplo de Iniciativa } \\
\text { (práticas na aeronáutica) }\end{array}$ & Autores \\
\hline $\begin{array}{l}\text { Trocar } \\
\text { - Substituir ○ antigo por } \\
\text { materiais não renováveis } \\
\text { avançados } \\
\text { - Aplicar novas tecnologias (por } \\
\text { exemplo, impressão 3D) } \\
\text { - Escolher novo produto/ serviço } \\
\text { (por exemplo, transporte } \\
\text { multimodal) }\end{array}$ & $\begin{array}{l}\text { - Design para desmontagem/ } \\
\text { reciclagem } \\
\text { - Design para modularidade } \\
\text { - Desenvolvimento de } \\
\text { competência para/e uso de } \\
\text { tecnologia ambientalmente } \\
\text { amigável } \\
\text { - Implementar novos } \\
\text { caminhos de sistemas } \\
\text { logísticos } \\
\text { - Transporte intermodal } \\
\text { - Foco na redução do } \\
\text { movimento de peças com } \\
\text { veículos }\end{array}$ & $\begin{array}{l}\text { KALMYKOVA, } \\
\text { SADAGOPAN E ROSADO, } \\
2018 \\
\text { KALMYKOVA, } \\
\text { SADAGOPAN E ROSADO, } \\
2018 \\
\text { AHI E SEARCY, } 2015 \\
\text { RẢDULESCU et al., } 2009 \\
\text { GOPALAKRISHNAN et al,, } \\
2012 \\
\text { GOVINDAN E HASANAGIC, } \\
2018 \\
\text { AHI E SEARCY, 2015 } \\
\text { VARSEI E POLYAKOVSKIY, } \\
2017 \\
\text { REEFKE E SUNDARAM, 2017 }\end{array}$ & $\begin{array}{l}\text { - Utilização de material sustentável para interiores de aeronaves. O painel } \\
\text { convencional é feito de um composto termofixo reforçado com fibra de vidro } \\
\text { com retardante de chama halogenado, enquanto os painéis sustentáveis são } \\
\text { feitos de polímeros renováveis ou recicláveis, reforços de fibras naturais e } \\
\text { retardadores de chama não halogenados. Além disso, os painéis sustentáveis } \\
\text { são mais leves que os painéis convencionais, a fim de reduzir o consumo de } \\
\text { combustivel e as emissões de poluentes atmosféricos da aeronave. Exemplos } \\
\text { de painéis sustentáveis: painel baseado em geopolímeros, painel de } \\
\text { biopolímero baseado em linseedoil e painéis termoplásticos com } \\
\text { polipropileno (PP) e com ácido polilático (PLA) }\end{array}$ & $\begin{array}{l}\text { SANTOS, LEIVA, COSTA } \\
\text { E GREGOLIN, } 2016 \\
\text { VIDAL et al., } 2017\end{array}$ \\
\hline
\end{tabular}

FIGURA 6 - Ação Trocar. Fonte: Autor (2019)

\section{DISCUSSÕES E CONSIDERAÇÕES FINAIS}

Como resultado final dessa pesquisa, foi proposto framework que possui potencial para iniciar discussões sobre a integração da economia circular no desenvolvimento de novos produtos, considerando especialmente as características dos produtos aeronáuticos.

Nos resultados das buscas foi possível observar que a energia renovável é, atualmente, um dos temas mais explorados pelas literaturas das áreas. Nesse sentido, esforços estão sendo feitos para melhorar o combustível de aviação que está sendo usado, o que tem o efeito de reduzir as emissões. Novos combustíveis alternativos, como aqueles produzidos a partir de materiais biológicos, podem reduzir drasticamente as emissões de $\mathrm{CO}_{2}$ da aviação, por isso, é tema amplamente investigado na área. Por exemplo, ao adaptar a qualidade do combustível ao design do motor, as aeronaves poderão produzir menos emissões por voo.

Outro tema relevante notado foi a remanufatura de produtos e componentes somada à reciclagem de materiais, práticas que têm crescido na indústria aeroespacial desde 2005. Interessante notar que o crescimento das investigações dessas práticas na indústria aeronáutica coincide com o período de crescimento das pesquisas sobre economia circular.

Porém, destaca-se que ainda são tímidas as iniciativas em implantar-se a manufatura sustentável na indústria aeronáutica. No setor aeronáutico, até poucos anos atrás, as aeronaves em fim de vida eram abandonadas em aterros sanitários em todo o mundo. A partir dos anos 
2000, os dois maiores fabricantes de aeronaves, a Airbus e a Boeing, começaram a desenvolver abordagens alternativas de como lidar com aeronaves no final de suas vidas. A Boeing fundou a associação setorial AFRA (Associação de Reciclagem de Frota de Aeronaves) juntamente com várias empresas de aviação e salvamento. Paralelamente aos esforços da Boeing para lidar com aeronaves em fim de vida, a Airbus, fabricante líder de aeronaves da Europa, lançou o projeto "Processo para Gerenciamento Avançado de Fim de Vida de Aeronaves" (PAMELA) em 2005 (RIBEIRO E GOMES, 2015).

Por fim, como todo trabalho de pesquisa, reconhece-se que este possui diversas limitações. A principal delas decorre de ter sido realizado apenas um esforço teórico. Como sugestão de futuras pesquisas, propõe-se, portanto, que o framework aqui apresentado seja avaliado por profissionais na área de desenvolvimento de produtos aeronáuticos. Isso ampliaria e melhoraria os resultados apresentados e discutidos neste artigo. A realização de estudo de casos múltiplos que apresentasse possibilidade da adoção da economia circular no desenvolvimento de produtos não apenas na indústria aeronáutica, mas em outras como a automobilística, de linha branca e eletrônica, por exemplo, também poderiam ser objeto de estudos futuros.

\section{AGRADECIMENTOS}

Os autores gostariam de agradecer o suporte da FAPESP (processo nº 18/23972-1).

\section{REFERÊNCIAS}

ABSON, D.J. et al. Leverage points for sustainability transformation. Ambio, v.46, p.1-10, 2017.

AHI, P., SEARCY, C. An analysis of metrics used to measure performance in green and sustainable supply chains. Journal of Cleaner Production, v.86, p.360-377, 2015.

ALLWOOD, J.M., ASHBY, M.F., GUTOWSKI, T.G., WORRELL, E. Material efficiency: a white paper. Resources, Conservation and Recycling, v.55, p.362-381, 2011.

ASMATULU, E., OVERCASH, M., TWOMEY, J.M. Evaluation of recycling efforts of aircraft companies in Wichita. Resources, Conservation and Recycling, v.80, p.36- 45, 2013.

AYGUN, H., TURAN, O. Entropy, Energy and Exergy for Measuring PW4000 Turbofan Sustainability. International Journal of Turbo and Jet Engines, 2019.

BAI, C., SARKIS, J. Green supplier development: analytical evaluation using rough set theory. Journal of Cleaner Production, v.18, p.1200-1210, 2010. 
BALLI O., SOHRET Y., KARAKOC H.T. The effects of hydrogen fuel usage on the exergetic performance of a turbojet engine. International Journal of Hydrogen Energy, v.43, p.10848-10858, 2018.

BAXTER G., SRISAENG P., WILD G. An assessment of airport sustainability, part 1-waste management at Copenhagen Airport. Resources, v.7, 2018.

BESSA L.C.B.A. et al. (Solid + liquid) equilibrium of binary mixtures containing ethyl esters and p-xylene by differential scanning calorimetry. Journal of Thermal Analysis and Calorimetry, 2019.

BESSETTE A.P. et al. Life Cycle Impacts and Techno-economic Implications of Flash Hydrolysis in Algae Processing. ACS Sustainable Chemistry and Engineering, v.6, p.3580-3588, 2018.

BOUKHERROUB T. et al. An integrated approach for sustainable supply chain planning. Computers \& Operations Research, v.54, 2014.

BWAPWA J.K., ANANDRAJ A., TROIS C. Microalgae processing for jet fuel production. Biofuels, Bioproducts and Biorefining, v.12, p.522-535, 2018.

CAVALETT O., CHERUBINI F. Contribution of jet fuel from forest residues to multiple Sustainable Development Goals. Nature Sustainability, v.1, p.799-807, 2018.

CETINKAYA B. et al. Sustainable supply chain management: Practical ideas for moving towards best practice. European Logistics Association, 2011.

CHEN L., REN J. Multi-attribute sustainability evaluation of alternative aviation fuels based on fuzzy ANP and fuzzy grey relational analysis. Journal of Air Transport Management, v.68, p.176-186, 2018.

COWPER-SMITH A., DE GROSBOIS, D. The adoption of corporate social responsibility practices in the airline industry. Journal of Sustainable Tourism, v.19, p.59-77, 2011.

DARNALL, N.; JOLLEY, G. J.; HANDFIELD, R. Environmental management systems and green supply chain management: complements for sustainability? Business Strategy and the Environment, v.17, p.30-45, 2008.

DINIZ A.P.M.M., SARGEANT R., MILLAR G.J. Stochastic techno-economic analysis of the production of aviation biofuel from oilseeds. Biotechnology for Biofuels, v.11, 2018.

DODD T., ORLITZKY M., NELSON T. What stalls a renewable energy industry? Industry outlook of the aviation biofuels industry in Australia, Germany, and the USA. Energy Policy, v.123, p.92-103, 2018.

EROL, I., SENCER, S., SARI, R. A new fuzzy multi-criteria framework for measuring sustainability performance of a supply chain. Ecological Economics, v.70, p.1088-1100, 2011.

EMF. Circular Economy Report - The Circular Economy - Towards a Circular Economy: Business Rationale for an Accelerated Transition, 2015. Ellenmacarthurfoundation.org [online] Disponível em: http://www.ellenmacarthurfoundation.org/publications/towards-a-circular-economy-business-rationale-for-anaccelerated-transition [Acesso em: 3 Mar. 2019].

EMF. The Circular Economy Concept - Regenerative Economy, 2017. Ellenmacarthurfoundation.org [online] Disponível em: https://www.ellenmacarthurfoundation.org/circular-economy/overview/concept [Acesso em: 2 Mar. 2019].

GIALOS, A.A. et al. Investigating the impact of sustainability in the production of aeronautical subscale components. Journal of Cleaner Production, v.176, p.785-799, 2018. 
GOPALAKRISH-NAN, K. et al. Sustainable supply chain management; A case study of Brirish Aerospace (BAE) Systems. International Journal Of Production Economics, 2012.

GOVINDAN, K., HASANAGIC, M. A systematic review on drivers, barriers, and practices towards circular economy: a supply chain perspective. International Journal of Production Research, v.56, p.278-311, 2018.

GRI (2012-2013) Sustainability Reporting Guidelines. Sustainability Reporting Guidelines.

HALLSTEDT, S.I. et al. Assessing sustainability and value of manufacturing processes: a case in the aerospace industry. Journal of Cleaner Production, v.108, p.169-182, 2015.

HENKE M. et al. Challenges and opportunities of very light high-performance electric drives for aviation. Energies, v.11, 2018.

HUANG, R. et al. Energy and emissions saving potential of additive manufacturing: the case of lightweight aircraft components. Journal of Cleaner Production, v.35, p.1559-1570, 2016.

IMMARIGEON, J.P. et al. Lightweight materials for aircraft applications. Materials Characterization, v.35, p. 41-67, 1995.

JI, P., MA, X., LI, G. Developing green purchasing relationships for the manufacturing industry: An evolutionary game theory perspective. International Journal of Production Economics, v.166, p.155-162, 2015.

KALMYKOVA Y., SADAGOPAN M., ROSADO L. Circular economy - From review of theories and practices to development of implementation tools. Resources, Conservation \& Recycling, v.135, p.190-201, 2018.

KEIVANPOUR, S., AIT KADI, D., MASCLE, C. End of life aircraft's recovery and green supply chain (a conceptual framework for addressing opportunities and challenges). Management Research Review, v.38, 2015. KIRCHOFF, J.F., KOCH, C., SATINOVER NICHOLS, B. Stakeholder perceptions of green marketing: the effect of demand and supply integration. International Journal of Physical Distribution \& Logistics Management, v.41, p.684-696, 2011.

KUHN H., FALTER C., SIZMANN A. Renewable Energy Perspectives for Aviation. CEAS - The International Conference of the European Aerospace Societies, 2011.

LIEDER, M., RASHID, A. Towards Circular Economy Implementation: A Comprehensive Review in Context of Manufacturing Industry. Journal of Cleaner Production, vol.115, p.36-51, 2016.

LINTON, J.D., KLASSEN, R., JAYARAMAN, V. Sustainable supply chains: An introduction. Journal of Operations Management, v.25, p.1075-1082, 2007.

MICHAILOS, S. Process design, economic evaluation and life cycle assessment of jet fuel production from sugar cane residue. Environmental Progress and Sustainable Energy, v.37, p.1227-1235, 2018.

MULDER, B., LA ROCCA, G., SCHUT, J., VERHAGEN, W.J.C. A Methodological Approach for the Optimisation of the Product Development Process by the Application of Design Automation. CEAS - The International Conference of the European Aerospace Societies, 2015. 
PARK, S., LEE, H., CHON, J. Sustainable monitoring coverage of unmanned aerial vehicle photogrammetry according to wing type and image resolution. Environmental Pollution, v.247, p.340-348, 2019.

PILLAIN, B., et al. Social life cycle assessment framework for evaluation of potential job creation with an application in the French carbon fiber aeronautical recycling sector. International Journal of Life Cycle Assessment, 2019.

RAJIANI, I., KOT, S. The prospective consumers of the Indonesian green aviation initiative for sustainable development in air transportation. Sustainability (Switzerland), v.10, 2018.

RAJ, A., SRIVASTAVA, S.K. Sustainability performance assessment of an aircraft manufacturing firm. Benchmarking, v.25, p.1500-1527, 2018.

RASSOULINEJAD-MOUSAVI, S.M., MAO, Y., ZHANG, Y. Reducing greenhouse gas emissions in Sandia methane-air flame by using a biofuel. Renewable Energy, v.128, p.313-323, 2018.

REEFKE, H., SUNDARAM, D. Key themes and research opportunities in sustainable supply chain management - identification and evaluation. Omega, vol.66, p.195-211, 2017.

RIBEIRO, J.S., GOMES, J.O. Proposed framework for End-Of-Life aircraft recycling. Procedia CIRP, v.26, p.311-316, 2015

SANTOS, C.V., LEIVA, D.R.,, COSTA, F.R., GREGOLIN, J.A.R. Materials Selection for Sustainable Executive Aircraft Interiors. Materials Research, v.19, p.339-352, 2016.

SARKER, F. et al. High-Performance Graphene-Based Natural Fiber Composites. ACS Applied Materials and Interfaces, v.10, p.34502-34512, 2018.

SMITH, A.D., OFFODILE, O.F. Green and sustainability corporate initiatives: a case study of goods and services design. International Journal of Process Management and Benchmarking, v.6, p.273-299, 2016.

SMOL, M. et al. The possible use of sewage sludge ash (SSA) in the construction industry as a way towards a circular economy. Journal of Cleaner Production, v.95, p.45-54, 2015.

SÖĞÜT, M.Z.. Exergetic Irreversibility and Sustainability Performances for Alternative Fuels in the MicroTurbojet Engine. International Journal of Green Energy, v.15, p.169-180, 2018.

SOUZA, C.S.R. et al. Reuse of Uncured Carbon Fiber/Epoxy Resin Prepreg Scraps: Mechanical Behavior and Environmental Response. ACS Sustainable Chemistry and Engineering, v.7, p.2200-2206, 2019.

SULLIVAN, J.L., LEWIS, G.M., KEOLEIAN, G.A.. Effect of mass on multimodal fuel consumption in moving people and freight in the U.S.. Transportation Research Part D: Transport and Environment, v.63, p.786808,2018

TUKKER, A.. Product Services for a Resource-efficient and Circular Economy - A Review. Journal of Cleaner Production, v.97, p.76-91, 2015

VACHON, S., KLASSEN, R.D. Environmental management and manufacturing performance: The role of collaboration in the supply chain. International Journal of Production Economics, v.111, p.299-315, 2008.

VARSEI, M., POLYAKOVSKIY, S. Sustainable supply chain network design: A case of the wine industry in Australia. Omega, v.66, p.236-247, 2017. 
VIDAL R. et al. Life Cycle Assessment of Novel Aircraft Interior Panels Made from Renewable or Recyclable Polymers with Natural Fiber Reinforcements and Non-Halogenated Flame Retardants. Journal of Industrial Ecology, v.22, p.132-144, 2018.

VINODH, S., RATHOD, G. Integration of ECQFD and LCA for sustainable product design. Journal of Cleaner Production, v.18, p. 833-842, 2010.

YAZAR, I. Simulation of a High Fidelity Turboshaft Engine-Alternator Model for Turboelectric Propulsion System Design and Applications. International Journal of Turbo and Jet Engines, 2018.

YIN, K.-S., WARD, A., DARGUSCH, P., HALOG, A.. The cost of abatement options to reduce carbon emissions from Australian international flights. International Journal of Sustainable Transportation, v.12, p.165-178, 2018.

ZHANG, N., XIE, H. Toward green IT: Modeling sustainable production characteristics for Chinese electronic information industry, 1980-2012. Technol. Forecast. Soc. Chang., v.96, p.62-70, 2014.

ZENG, D., FU, X., OUYANG, T. Implementing Green IT Transformation for Sustainability: A Case Study in China. Sustainability, v.10, 2018.

ZHU, Q., GENG, Y., LAI. K. H. Circular Economy Practices among Chinese Manufacturers Varying in Environmental-oriented Supply Chain Cooperation and the Performance Implications. Journal of Environmental Management, v.91, p.1324-1331, 2010.

ZHU, Q., SARKIS, J. Relationships Between Operational Practices and Performance Among Early Adopters of Green Supply Chain Management Practices in Chinese Manufacturing Enterprises. Journal of Operations Management, v.22, p.265-289, 2004. 